\title{
The relationship between Anti-Mullerian Hormone (AMH) levels and pregnancy outcomes in patients undergoing assisted reproductive techniques (ART)
}

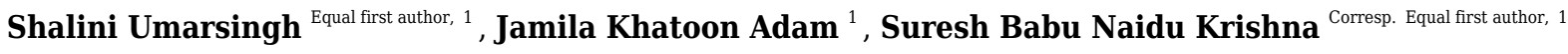 \\ 1 Biomedical and Clinical Technology, Faculty of Health Sciences, Durban University of Technology, Durban, South Africa \\ Corresponding Author: Suresh Babu Naidu Krishna \\ Email address: Sureshk@dut.ac.za
}

A variety of predictors are available for ovarian stimulation cycles in assisted reproductive technology (ART) forecasting ovarian response and reproductive outcome in women including biomarkers such as anti-Mullerian hormone (AMH). The aim of our present study was to compare the relationship between AMH levels and pregnancy outcomes in patients undergoing intra-cytoplasmic sperm injection (ICSI). Overall, fifty patients $(n=50)$, aged 20-45 years were recruited for the present prospective study. Three AMH levels were presented with high often poly cystic ovarian syndrome (PCOS) amongst $52.4 \%$ patients, $40.5 \%$ in normal and $7.1 \%$ in low to normal, correspondingly. There was statistically significant relationship between AMH and day of embryo transfer $(p<0.05)$. The Pearson analysis between $\mathrm{AMH}$, age, E2 and FSH displayed no statistically significant relationship between E2 and AMH $(p<0.05)$ and negative correlation between FSH and age $(p>0.05)$. The area under the receiver operating characteristic curve for $E_{2}$ was 0.725 and for $A M H$ levels as predictors of CPR was 0.497 indicating $E_{2}$ as better predictor than $\mathrm{AMH}$. The number of oocytes, mature oocytes and fertilized oocytes all presented a weak positive relationship to $\mathrm{AMH}$. Our results confirm the clinical significance of $\mathrm{AMH}$ to accurately predict ovarian reserve as a marker and its limitations to use as predictor for a positive pregnancy outcome. Additional prospective studies should be conducted to validate the predictive capability of AMH levels for the outcome of clinical pregnancy. 
1 Title

2 The Relationship Between Anti-Mullerian Hormone (AMH) Levels and 3 Pregnancy Outcomes in Patients Undergoing Assisted Reproductive 4 Techniques (ART)

Authors Affiliations

1. Shalini Umarsingh, Department of Biomedical \&Clinical Technology, Faculty of Health Sciences, Durban University of Technology, Durban-4000, South Africa Email: $\underline{\text { shalini.umarsingh@gmail.com; }}$

2. Prof Jamila Khatoon Adam, Department of Biomedical \&Clinical Technology, Faculty of Health Sciences, Durban University of Technology, Durban-4000, South Africa Email: adamjk@dut.ac.za

3. Dr Suresh Babu Naidu Krishna, Department of Biomedical \&Clinical Technology, Faculty of Health Sciences, Durban University of Technology, Durban-4000, South Africa Email: sureshk@dut.ac.za

*Corresponding author

Word Count: 5001 
Abstract

28

29

A variety of predictors are available for ovarian stimulation cycles in assisted reproductive technology (ART) forecasting ovarian response and reproductive outcome in women including biomarkers such as anti-Mullerian hormone (AMH). The aim of our present study was to compare the relationship between AMH levels and pregnancy outcomes in patients undergoing intracytoplasmic sperm injection (ICSI). Overall, fifty patients $(n=50)$, aged 20-45 years were recruited for the present prospective study. Three AMH levels were presented with high often poly cystic ovarian syndrome (PCOS) amongst 52.4\% patients, 40.5\% in normal and 7.1\% in low to normal, correspondingly. There was statistically significant relationship between AMH and day of embryo transfer $(p<0.05)$. The Pearson analysis between AMH, age, E2 and FSH displayed no statistically significant relationship between E2 and AMH $(p<0.05)$ and negative correlation between FSH and age $(p>0.05)$. The area under the receiver operating characteristic curve for $\mathrm{E}_{2}$ was 0.725 and for $\mathrm{AMH}$ levels as predictors of CPR was 0.497 indicating $\mathrm{E}_{2}$ as better predictor than AMH. The number of oocytes, mature oocytes and fertilized oocytes all presented a weak positive relationship to $\mathrm{AMH}$. Our results confirm the clinical significance of $\mathrm{AMH}$ to accurately predict ovarian reserve as a marker and its limitations to use as predictor for a positive pregnancy outcome. Additional prospective studies should be conducted to validate the predictive capability of AMH levels for the outcome of clinical pregnancy.

Keywords: Anti-Mullerian hormone, Fertilization in vitro, Pregnancy, Enzyme-linked immunosorbent assay, Oocytes, Clinical pregnancy rate 


\section{Introduction}

56

Couples in modern societies postpone childbearing amidst busy schedules and career positionings; trying to conceive at a more advanced age contributing to a rise in the occurrence of infertility (Caroppo et al., 2006). Most women are unaware that fertility starts to decline after the early thirties in some individuals. With increasing female age, fecundity in natural and stimulated ovarian cycles declines, as observed in population-based studies (Grynnerup et al., 2012) as well as in IVF studies (Scheffer et al., 2018). For this reason, there is a growing number of women of advanced age seeking treatment for infertility (Oskayli et al., 2019).

The hormonal control of ovarian function is influenced by administering exogenous follicle stimulating hormone (FSH) (Richards, 2018). Prediction of ovarian responses prior to stimulation is not only useful for patient counselling, but also important in tailoring the optimal dosage of gonadotrophin for individual patients. The recruitment and development of multiple follicles in response to gonadotrophin stimulation are essential for the successful treatment of infertility by assisted reproductive techniques (ART) (Dewailly et al. 2014; Yang, Wu, and Zhang 2020). Besides, poor ovarian response has been suggested to be associated with high cycle cancellation rates (Saldeen, Källen, and Sundström 2007). Chang et al (1998) found that patients with antral follicle number $\leq 3$ had a significantly higher rate of cycle cancellation and higher human menopausal gonadotropin (HMG) dosage as compared with those patients with antral follicle number $4-10$ or $\geq 10$. Nevertheless, the AFC is presently believed to be the finest specific predictor of ovarian response to stimulation in ART, and it can be used in clinical practice for pretreatment counselling targets.

In assisted reproduction, serum levels for several hormones are used to assess the ovarian reserve and to monitor the development of the follicles that have been stimulated by gonadotrophins(Alson et al., 2018). Traditional techniques used to predict ovarian stimulation have incorporated serum levels of hormones such as FSH, LH and estrogen $\left(\mathrm{E}_{2}\right)$ along with ultrasonographic guides such as ovarian volume and number of early antral follicles as a reliable predictor of the outcome of in vitro fertilization (IVF) (Kunt et al., 2011). Over the last years, Anti-Mullerian hormone (AMH) 
82 has been projected as a novel marker for predicting ovarian response to gonadotrophin stimulation 83 (Alson et al., 2018, Zargar et al., 2018). AMH is a dimeric glycoprotein strongly produced by the

84 granulosa cells of the pre-antral (primary and secondary) and small antral follicles (AF's) in the ovary and shown to be age dependent (Sahmay et al., 2014). Measurement of anti-Müllerian hormone in serum is much more precise measure of the ovarian reserve than the other hormones that have previously been available to us(Anderson et al., 2012).

The aim of this prospective study was to investigate the relationship between Anti-Müllerian hormone levels and pregnancy outcomes in patients undergoing in-vitro fertilization or intracytoplasmic sperm injection (ICSI).

\section{Materials and Methods}

Patients: Fifty women(n=50), aged 20-45 years were recruited from Centre of Assisted Reproduction and Endocrinology (C.A.R.E) Clinic in Westville, Durban, South Africa who were undergoing IVF treatment. This study was approved by Ethical Committee of the Durban University of Technology (Project reference 128/16) and Research Committee, C.A.R.E. Clinic, Durban, South Africa. After approving the study by the research ethics committees, written informed consents were obtained from all the patients.

GnRH antagonist Protocol: A gonadotrophin-releasing hormone (GnRH) antagonist protocol with recombinant FSH (GONAL-f, Merck Serono, Darmstadt, Germany) was used as downregulatory (Park et al., 2015). The second approach was followed by administering 0.25 mg/day Cetrotide (Merck Serono). When at least 3 or more follicles reach a diameter equal or above $17-18 \mathrm{~mm}$, the endometrial thickness reached at least $7 \mathrm{~mm}$ by ultrasound and E2 levels were about 1500-1800 pmol/L then Human chorionic gonadotropin (hCG) was administered. All patients received $5000-10000 \mathrm{IU}$ hCG (Ovitrelle ${ }^{\circledR}$, Merck Serono). Oocyte retrieval was performed 36 hours after the administration of the hCG. Conventional IVF or ICSI was performed according to previously described protocols.

Sample collection: Blood samples were collected every 3-4 days on commencement of the treatment. The blood samples were centrifuged at $3000 \mathrm{rpm}$ for 10 minutes using a Biofuge centrifuge (Biofuge Primo - Heraeus) to obtain the blood serum. AMH and FSH levels were 
111 recorded, upon the first visit. Estrogen $\left(E_{2}\right)$ and LH levels were monitored throughout the program 112 until a peak $\mathrm{E}_{2}$ and $\mathrm{LH}$ level were reached.

113 Hormone Assays: Gen II ELISA (Beckman Coulter Inc., USA, catalog number A79765/A79766, unmodified version). (Beckman Coulter, USA) kit was used to estimate hormone levels (FSH-Cat. No. 33520 Access hFSH reagent, 100 determinations, 2 x 50 tests); E2 (Cat. No. B84493 Access Sensitive Estradiol Reagent, 100 determinations) and AMH (Cat. No. B13127 Access AMH Reagent, 100 determinations, 2 x 50 tests) from the blood serum according to manufacturer's instructions. Insemination and intra cytoplasmic sperm injection (ICSI), oocyte retrieval, culture, fertilization, embryo culture, and transfer were carried out as previously described by Gardener et al. (Gardner et al., 2001).

Inclusion Criteria: The population of the study included female patients ranging between the ages of $20-45$

Exclusion Criteria: Patients undergoing cancer therapy and patients on immune suppressant drugs were excluded from study.

Statistical analysis: The data were analysed using IBM SPSS software (Chicago, IL, USA). Pearson's correlation was used to determine the direction, strength, and significance of the correlation between $\mathrm{X}$ and $\mathrm{Y}$ variables between the different semen parameters. A parametric multiple linear regression analysis was used to evaluate the relationship between AMH and other available endocrine markers. ROC curves were used to assess predictive value for $\mathrm{E}_{2}$ and $\mathrm{AMH}$ and evaluating cut off values to optimise sensitivity and specificity. A $p$ value of $<0.05$ was considered statistically significant.

Institutional Review Board approval: This study was approved by Ethical Committee of the Durban University of Technology (Project reference 128/16) and Research Committee, C.A.R.E. 1975 (as revised in 1983).

\section{Results}

137 The prospective study included fifty patients who met the inclusion criteria. From the initial sample 138 size of fifty, forty-two presented with data that could be analysed whilst 8 patients had oocytes 
139 that where abnormal and did not result in transfer. The data from these 8 patients were not included

140 in the study due to poor embryo development (Table 1).

141 Table 1. AMH distribution in blood samples

142 Amongst the 42 patients analysed, 4.76\% were between 20-24 years, 9.52\% were between 25-29

143 years, 40.47\% were between 30-34 years, 35.7\% were between 35-39 years and 9.52\% were

144 between 40-44 years, respectively. As demonstrated by this study the clinical pregnancy rate for

145 patients 20 - 24 years was $100 \%, 25-29$ years was $50 \%, 30-34$ years was $17.6 \%, 35$ - 39 years

146 was $26.6 \%$ and $40-44$ years was $25 \%$ (Fig. 1 ).

147 Table 2 shows number of oocytes retrieved, number of oocytes matured, and number of oocytes

148 fertilized into respective categories. Not all eggs obtained were at the metaphase 2 stages and had

149 to be matured in the incubator overnight and injected the following day. The results shown were

150 to some extent anticipated as $\mathrm{AMH}$ has been used an indicator of oocyte reserve in previous

151 studies(Yarde et al., 2013, Yao et al., 2015) whereas the resulting fertilized or transferred embryo's

152 may be due to a chance process based on many various factors such as quality of oocyte and 153 sperm.

154 The Chi-square test for Independence was performed to check whether there was an association 155 between the number of oocytes fertilized and the AMH category (Table 2). A Chi-squared value 156 of 18.5 , degrees of freedom $=12$, with a $p=0.10$ was found. There was no statistically significant 157 relationship between numbers of oocytes fertilized versus AMH category $(p>0.05)$.

158 Out of 22 patients, 43 embryos were transferred. Embryos were transferred depending on embryo 159 development and the number of embryos obtained. Most patients in the high and normal categories 160 resulted in a day 5 transfer, the Chi-squared test for independence of AMH and number of embryos 161 transferred gave a Chi-squared value of 6.384 with $\mathrm{df}=4$ and $p$-value $=0.172$ (Table 3 ), thus 162 statistically no significant association between AMH and number of embryos transferred was 163 observed. Whilst, Chi-square test for independence between the variables AMH and day of embryo 164 transfer (Table 4) gave a Chi-square value of 14.117, 6 degrees of freedom and $p=0.028$ indicating 165 statistically significant relationship between AMH and day of embryo transfer $(p<0.05)$.

166 Pregnancy outcome and AMH category are as shown in Table 5. Out of twenty two cases in high 167 category, 6 resulted in a positive pregnancy; 6 resulted in a positive outcome $(6 / 12=50.0 \%)$ 
168 (Normal); while out of the 3 cases where the AMH was "Low to Normal", there were no 169 pregnancies reported. The Chi-squared test for independence of AMH category and pregnancy 170 outcome gave a Chi-Squared value of $0.502,2$ degrees of freedom and $p=0.778$. There was no

171 statistically significant relationship between the pregnancy outcome and the AMH category $(p>$ $1720.05)$.

\section{Pearson correlation}

174 Pearson correlation coefficients were calculated to determine if any statistical significance exists 175 between $\mathrm{AMH}$ on a quantitative scale and age, $\mathrm{E}_{2}$ and FSH (Table 6). The Pearson Correlation co176 efficient of 0.151 indicates that a very weak positive relationship existence between $\mathrm{E}_{2}$ and $\mathrm{AMH}$, 177 which is not statistically significant $(p=0.341)$. Furthermore, Pearson correlation coefficient 178 between the AMH and age had a coefficient of -0.028 thus showing no statistical significance $p=$ $1790.859(p>0.05)$. The Pearson Correlation between AMH and FSH produced a coefficient of 1800.185 thus indicating no statistical significance $p=0.240(p>0.05)$. Pearson correlation coefficient 181 showed no significant association between AMH and number of oocytes $(p=0.191)$, number of 182 mature oocytes $(p=0.300)$ and number of oocytes fertilized $(p=0.146)$. The number of oocytes, 183 mature oocytes and oocytes fertilized all presented a no statistically significant correlation with $184 \operatorname{AMH}(0.206,0.164$, and 0.228 , respectively).

185 Logistic regression analysis

186 A logistic regression model was used to determine the possible predictor variables for the 187 pregnancy outcome. The model was fitted to the data with the result of the pregnancy namely, 188 189 190 191 192

Area under the curve

195

The ROC curves of the serum $\mathrm{AMH}$ concentrations and $\mathrm{E}_{2}$ for the prediction of the clinical 196 pregnancy are depicted in Fig. 2. The areas under the curves (AUC) for $E_{2}$ were 0.725 and for 
$197 \mathrm{AMH}(\mathrm{AUC}=0.497) . \quad \mathrm{E}_{2}$ is therefore a better single predictor of pregnancy outcome when

198 compared to AMH. It has been shown that $\mathrm{E}_{2}$ can better predict the number of oocytes obtained.

\section{Discussion}

200 In the current study, we investigated the relationship between AMH levels and pregnancy 201 outcomes in patients undergoing intra-cytoplasmic sperm injection.

202

203

204

205

206

207

208

209

210

211

212

213

214

215

216

217

218

219

220

221

222

223

224

225

Correlation between basal $A M H$ and $E_{2}$

Pearson analysis between $\mathrm{E}_{2}$ and $\mathrm{AMH}$ presented a Pearson Correlation co-efficient of 0.151 with $p=0.341(p<0.05)$ which indicates that a weak significant relationship exists between $\mathrm{E}_{2}$ and AMH. Most previous studies (Ramalho de Carvalho et al., 2012, Ubaldi et al., 2005) have shown a relationship between a raised basal $\mathrm{E}_{2}$ level and a reduced ovarian response using different values to express elevated estrogen levels which replicated the findings in this study therefore showing that a low AMH can result in low estrogen levels. Also, it can be concluded that a poor $\mathrm{AMH}$ value results in a poor ovarian reserve indicating follicles produced will not be correlated to a raised estrogen level, therefore indicating poor follicle growth, thus reducing the number of oocytes produced. However, it was determined that meagre response to stimulus in IVF, indicative of a lower ovarian reserve, is associated with declined baseline serum AMH concentrations (Van Rooij et al., 2004). Consequently, when women have regular ovarian reserve and decent retort, disappointment of IVF must look for additional infertility reasons, e.g., male specific issue i.e. Y chromosome microdeletion. Furthermore, this conclusion is reinforced by the data of woman undergoing IVF which indicated that male factor infertility resulted in an unsuccessful cycle. Although E2 levels in these cases were above those of controls, they are still within the range of 25-100 pg/ml (Sahmay et al., 2014), suggesting that E2 single-handedly is not capable of predicting the female reproductive potential.

\section{Correlation between basal AMH and Age}

Pearson correlation between AMH and age (Table 6) presented a co-efficient of -0.028 thus displaying a weak, negative association with a $p=0.859(p>0.05)$. A stronger relationship between these two variables was expected as it is known that as age increases, AMH should decrease. This contrary association corresponds as reported by Van Rooij et al., (2004), where serum AMH levels decline with age in normal women with proven fertility. Also, it is suggested that serum AMH is 
226 identified as the improved endocrine marker to measure the reproductive capability in advanced 227 age.

228

229

230

231

232

233

234

235

236

237

238

239

240

241

242

243

244

245

246

247

248

249

250

251

252

253

254

\section{Correlation between Basal AMH and FSH}

Basal FSH is one of the primary endocrine markers presented into ART program. The Pearson correlation amid AMH and FSH (Table 6) had a coefficient of -0.185 thus displaying a weak, negative relationship and with a $p=0.240(p>0.05)$. This study specifies a negative correlation, i.e., the higher the FSH the higher the chances the patient can present with a poor ovarian reserve and early menopause. It is routine practice to frequently measure the basal FSH level and to start IVF treatment only when the FSH level is lower than threshold value in a cycle. This was strategic on the awareness that these women will retort well to ovarian stimulation while the basal FSH level is lesser at the beginning of the cycle. The outcomes of this study revealed that woman who were poor respondents or had a reduced ovarian reserve had a poor outcome and frequent testing is of no worth. Women who had a history of high FSH level must undergo treatment without further delay. By postponing treatment for these patients can be detrimental as they get older and fast approaching menopause (Uzumcu and Zama, 2016).

\section{Correlation between Age and FSH and Number of oocytes}

The Pearson Correlation between FSH and age (Table 6) displayed no statistical significance, $p=$ $0.583(p>0.05)$. For most of Pearson Correlation analysis, no significant relationships were found with most of the $p$-values, being greater than 0.05 . This may be due to the small sample size used in this study of 42 patients. AMH being compared to age and number of oocytes showed a slightly negative correlation which is expected as it is shown in previous studies that $\mathrm{AMH}$ and number of oocytes decrease with maternal age (van Rooij et al., 2004) and Gobikrushant et al. (2018). This inverse relationship is in agreement by Van Rooij et al.

(2004), who reported that serum $\mathrm{AMH}$ levels deteriorate with age in normal women with proven fertility. Additionally, serum AMH indicates the simplest endocrine marker to measure the age-related decline of reproductive competence. AMH levels, in our group who were high respondents were over $3.0 \mathrm{ng} / \mathrm{ml}$, normal respondent over $1.0 \mathrm{ng} / \mathrm{ml}$ and low respondents found to be below $0.9 \mathrm{ng} / \mathrm{ml}$. Oocytes were still recovered even with low AMH levels. Neither fertilization rate nor embryo quality can be assessed using basal AMH levels. This contrasts with the findings reported by Vaegter et al. (2017), where 
255 embryos had superior morphology and cleavage performance in patients with AMH levels $>2.7$

$256 \mathrm{ng} / \mathrm{ml}$ as compared with patients with values below this threshold.

257 Correlation between AMH and the number of oocytes, number of mature oocytes and number of 258 oocytes fertilized

259 Our second objective of the study was to examine if AMH levels affected oocyte quality. In this 260 study, the Pearson Correlation test (Table 6) showed no significant relationship between AMH and 261 number of oocytes ( $p=0.191)$, several mature oocytes $(p=0.300)$ and number of oocytes fertilized $262(\mathrm{p}=0.146)$. The number of oocytes, mature oocytes and oocytes fertilized all showed a weak 263 positive relationship to $\mathrm{AMH}(0.206,0.164$, and 0.228 , respectively). These findings are in 264 agreement with that reported by La Marca and Sunkara (2014); La Marca et al., (2010) and 265 Dehghani-Firouzabadi et al. (2008), where mean amount of oocytes was lower in poor responding 266 patients than in normal patients attending IVF programs. This therefore led to the inference that 267 ovarian response can be regarded as a reflection of the ovarian reserve. The Chi-square test for 268 Independence was done to determine whether there is an association between the number of 269 oocytes collected and the AMH category (Table 8). A Chi-squared value of 21.246, degrees of 270 freedom $=8$, with a $p=0.007$ was observed. There was a significant relationship between the numbers of oocytes collected versus AMH category $(p<0.05)$. The Chi-square test for

272 Independence was performed to see whether there is an association between the number of oocytes

273 fertilized and the AMH category (Table 9). A Chi-squared value of 18.5, degrees of freedom = 12, 274 with a $p=0.10$ was found. There was thus no statistically significant relationship between the 275 numbers of embryo's fertilized versus AMH category $(p>0.05)$. This is anticipated as AMH has 276 been used an indicator of oocyte reserve in previous studies whereas the resulting fertilized or 277 transferred embryo's may be due to a chance process based on many various factors such as the 278 quality of the oocyte and sperm (Yarde et al., 2013). Ebner et al. (2006), demonstrated that AMH 279 serum levels were related with oocyte quality in stimulated cycles. The quality of the embryos was 280 not assessed using baseline AMH which agrees with our findings. However, the fertilization rate 281 was not correlated with the serum AMH which varied with the results of the present study.

\section{AMH category and positive pregnancies}

283 Embryo quality has been suggested to be of paramount importance to predict the occurrence of 284 pregnancy after IVF. In a regression model $\mathrm{E}_{2}$ has a $p=0.017(p<0.05)$ and LH has a $p=0.035$ 
$285(p<0.05)$. Both variables are significant and age and basal AMH play a role in the pregnancy

286 outcome and the model is thus adjusted for these two variables

287 In this study, AMH value for predicting pregnancy outcomes does not exist because oocyte quality 288 is not accounted for by ovarian reserve markers. As demonstrated in this study the clinical 289 pregnancy rate for patients 20 - 24 years was $100 \%, 25$ - 29 was 50\%, 30 - 34 years was 18\%, 35 $290-39$ years was $27 \%$ and 40 - 44 years was 25\% (Figure 1). Patients presenting with a low AMH 291 did not vary from those women presenting with higher AMH concentrations in same age group. A 292 positive pregnancy outcome was logged across all age groups regardless of the AMH level. These 293 results advocate that low ovarian reserve is not correlated with low oocyte quality in patients and 294 the prediction remains the similar despite low AMH concentrations. Kini et al. (2010) stated the 295 role of AMH in foreseeing cumulative pregnancy outcome during IVF treatment. It was recognized 296 that serum AMH concentration on day 6 of stimulation was suggestively higher in participants 297 who resulted in an ongoing pregnancy in IVF compared to those who did not. Serum AMH is a 298 suitable indicator of ovarian hyper-response. In a metanalysis study conducted by Yao et al. (2015) 299 to evaluate role of serum AMH role in forecasting the pregnancy outcome in IVF/ICS, it was 300 concluded that there is positive correlation between serum AMH and pregnancy. Nevertheless, 301 association between serum AMH and non-pregnancy cannot be ruled out either.

\section{Conclusion}

303 In conclusion, the outcomes of our investigations specify that AMH has established to be a 304 valuable marker for ovarian reserve and might benefit woman who plan for pregnancy. AMH 305 hormone seems to be the best endocrine marker, however, the valuable role of AMH and its role 306 in ovarian function should be looked at in relation to the other markers to assess the decline of the 307 ovarian pool. While appropriate reference values are being generated per age category and until 308 the consequences of having a low or high AMH for one's age are being established, AMH should 309 only be determined in the context of clinical studies. At present, the most important clinical role 310 of AMH at this stage is to serve as a red flag for reduced ovarian reserve in women of reproductive 311 age who must undergo further diagnostics. As per the study conducted, we can infer that AMH 312 can accurately predict ovarian reserve but cannot predict the oocyte quality or a positive pregnancy 313 outcome. The more oocytes obtained, increases a patient's chance of more viable embryos and 314 therefore, improving chances of a healthy pregnancy and ultimately a live birth. Further research 
315 on the implication of varying levels of AMH within the follicular fluid may be representative as

316 an indicator of "quality" in addition to the number of growing follicles.

\section{Study Limitation}

318 A noteworthy restraint of the current study was the lack of antral follicle count (AFC) at time of oocyte collection.

Conflict of Interest

Authors declare no conflict of interest

322

323

324

325

\section{Authors Contribution}

$[\mathrm{SU}]$ and $[\mathrm{JKA}]$ contributed to the study conception and design. [SU] performed all experiments, [SU], [KSBN] and [JKA] performed material preparation, data collection and analysis. The first draft of the manuscript was written by [KSBN] and all authors commented on previous versions of the manuscript. All authors read and approved the final manuscript.

\section{References}

Alson SSE, Bungum IJ, Giwercman A \& Henic E. 2018. Anti-mullerian hormone levels are associated with live birth rates in ART, but the predictive ability of anti-mullerian hormone is modest. European Journal of Obstetrics \& Gynecology and Reproductive Biology, 225, 199-204.

Anderson RA, Nelson SM \& Wallace WHB. 2012. Measuring anti-Müllerian hormone for the assessment of ovarian reserve: When and for whom is it indicated? Maturitas, 71, 28-33.

Chang, M.Y., Chiang, C.H., T'sang-T'ang Hsieh, M.D., Soong, Y.K. and Hsu, K.H., . 1998. 'Use of the antral follicle count to predict the outcome of assisted reproductive technologies. ', Fertility and Sterility, 69, 505-510

Caroppo E., Matteo M, Schonauer IM, Vizziello G, Pasquadibisceglie A, Vitti A \& Damato G. 2006. Basal FSH concentration as a predictor of IVF outcome in older women undergoing stimulation with GnRH antagonist. Reproductive biomedicine online, 13, 815-820.

Dehghani FR, Tayebi, N \& Asgharnia M. 2008. Serum level of anti-mullerian hormone in early follicular phase as a predictor of ovarian reserve and pregnancy outcome in assisted reproductive technology cycles.

Dewailly, Didier, Claus Yding Andersen, Adam Balen, Frank Broekmans, Nafi Dilaver, Renato Fanchin, Georg Griesinger, Tom W Kelsey, Antonio La Marca, and Cornelius Lambalk. 2014. 'The physiology and clinical utility of anti-Müllerian hormone in women', Human Reproduction Update, 20: 37085. 
Ebner T, Sommergruber M, Moser M, Shebl O, Schreier-lechner E \& Tews G. 2006. Basal level of antiMüllerian hormone is associated with oocyte quality in stimulated cycles. Human reproduction, 21, 2022-2026.

Gardner DK, lane M, Stevens J \& Schoolcraft WB. 2001. Noninvasive assessment of human embryo nutrient consumption as a measure of developmental potential. Fertility and sterility, 76, 11751180.

Gobikrushanth M, Purfield DC, Colazo M, Butler ST, Wang Z. \& Ambrose D. 2018. The relationship between serum anti-Müllerian hormone concentrations and fertility, and genome-wide associations for anti-Müllerian hormone in Holstein cows. Journal of dairy science, 101, 7563-7574.

Grynnerup AGA, Lindhard A \& Sorensen S. 2012. The role of anti-Mullerian hormone in female fertility and infertility - an overview. Acta Obstetricia Et Gynecologica Scandinavica, 91, 1252-1260.

Kini S, Li HR, Morrell, D, Pickering S \& Thong KJ. 2010. Anti-mullerian hormone and cumulative pregnancy outcome in in-vitro fertilization. Journal of assisted reproduction and genetics, 27, 449-456.

Kumar A, Kalra B, Patel A, McDavid I \& Roudebush WE. 2010. Development of a second generation antiMüllerian hormone (AMH) ELISA. Journal of immunological methods, 362, 51-59.

Kunt C, Ozaksit G, Kurt RK, Gungor ANC, Kanat-Pektas M, Kilic S \& Dede A. 2011. Anti-Mullerian hormone is a better marker than inhibin B, follicle stimulating hormone, estradiol or antral follicle count in predicting the outcome of in vitro fertilization. Archives of Gynecology and Obstetrics, 283, 14151421.

La Marca A, Sighinolfi G, Radi D, Argento,C, Baraldi E, Artenisio AC, Stabile G \& Volpe A. 2010. AntiMullerian hormone (AMH) as a predictive marker in assisted reproductive technology (ART). Human Reproduction Update, 16, 113-130.

La Marca A \& Sunkara SK. 2014. Individualization of controlled ovarian stimulation in IVF using ovarian reserve markers: from theory to practice. Human Reproduction Update, 20, 124-140.

Oskayli MC, Gulcin N, Ozatman E, Gercel G, Mutus M, Aksu B \& Durakbasa CU. 2019. Assessment of ovarian reserve using serum anti-Mullerian hormone after ovarian torsion surgery. Pediatrics International, 61, 504-507.

Palermo R. 2007. Differential actions of FSH and LH during folliculogenesis. Reproductive biomedicine online, 15, 326-337.

Park HJ, lyu SW, Seok HH, Yoon TK \& Lee WS. 2015. Anti-Müllerian hormone levels as a predictor of clinical pregnancy in in vitro fertilization/intracytoplasmic sperm injection-embryo transfer cycles in patients over 40 years of age. Clinical and Experimental Reproductive Medicine, 42, 143.

Ramalho de Carvalho B, Gomes Sobrinho DB, Vieira ADD, Resend MPS, Barbosa ACP, Silva AA \& nakagava, h. m. 2012. Ovarian reserve assessment for infertility investigation. ISRN obstetrics and gynecology, 2012.

Richards JS. 2018. The ovarian cycle. Vitamins and hormones. Elsevier.

Saldeen, Pia, Karin Källen, and Per Sundström. 2007. 'The probability of successful IVF outcome after poor ovarian response', Acta Obstetricia Et Gynecologica Scandinavica, 86: 457-61

Sahmay S, Oncul M, Tuten A, Tok A, Acikgoz AS \& Cepni I. 2014. Anti-Müllerian hormone levels as a predictor of the pregnancy rate in women of advanced reproductive age. Journal of assisted reproduction and genetics, 31, 1469-1474.

Scheffer JAB, Scheffer B, Scheffer R, Florencio F, Grynberg M \& Lozano DM. 2018. Are age and antiMullerian hormone good predictors of ovarian reserve and response in women undergoing IVF? Jornal Brasileiro De Reproducao Assistida, 22, 215-220.

Ubaldi FM, Rienzi I, Ferrero S, Baroni E, Sapienza F, Cobellis I \& Greco E. 2005. Management of poor ovarian responders in IVF. Reproductive biomedicine online, 10, 235-246.

Uzumcu M \& Zama AM. 2016. Developmental Effects of Endocrine-Disrupting Chemicals in the Ovary and on Female Fertility. The Epigenome and Developmental Origins of Health and Disease. Elsevier.

Peer) reviewing PDF | (2020:08:52172:2:0:NEW 22 Oct 2020) 
Vaegter KK, Lakic TG, Olovsson M, Berglund I, Brodin T \& Holte J. 2017. Which factors are most predictive for live birth after in vitro fertilization and intracytoplasmic sperm injection (IVF/ICSI) treatments? Analysis of 100 prospectively recorded variables in 8,400 IVF/ICSI single-embryo transfers. Fertility and sterility, 107, 641-648. e2.

Van Rooij IA, Den Tonkelaar I, Broekmans FJ, Looman CW, Scheffer GJ, de Jong FH, Themmen AP \& Te Velde ER. 2004. Anti-müllerian hormone is a promising predictor for the occurrence of the menopausal transition. Menopause, 11, 601-606.

Yao I, Zhang W, Li H \& Lin W. 2015. The role of serum AMH and FF AMH in predicting pregnancy outcome in the fresh cycle of IVF/ICSI: a meta-analysis. International journal of clinical and experimental medicine, 8, 1755.

Yang, Peiwen, Ruxing Wu, and Hanwang Zhang. 2020. 'The effect of growth hormone supplementation in poor ovarian responders undergoing IVF or ICSI: a meta-analysis of randomized controlled trials', Reproductive Biology and Endocrinology, 18: 76.

Yarde F, Voorhuis M, Dolleman M, Knauff EAH, Eijkemans MJC \& Broekmans FJM. 2013. Antimullerian hormone as predictor of reproductive outcome in subfertile women with elevated basal folliclestimulating hormone levels: a follow-up study. Fertility and Sterility, 100, 831-+.

Zargar M, Najafian M \& Zamanpour Z. 2018. Relationship between follicular fluid and serum anti-Mullerian hormone levels and pregnancy rate in ART cycles. Perinatología y Reproducción Humana, 32, 3-8. 
Figure 1 (on next page)

Number of patients associated with pregnancies in relevant age groups 


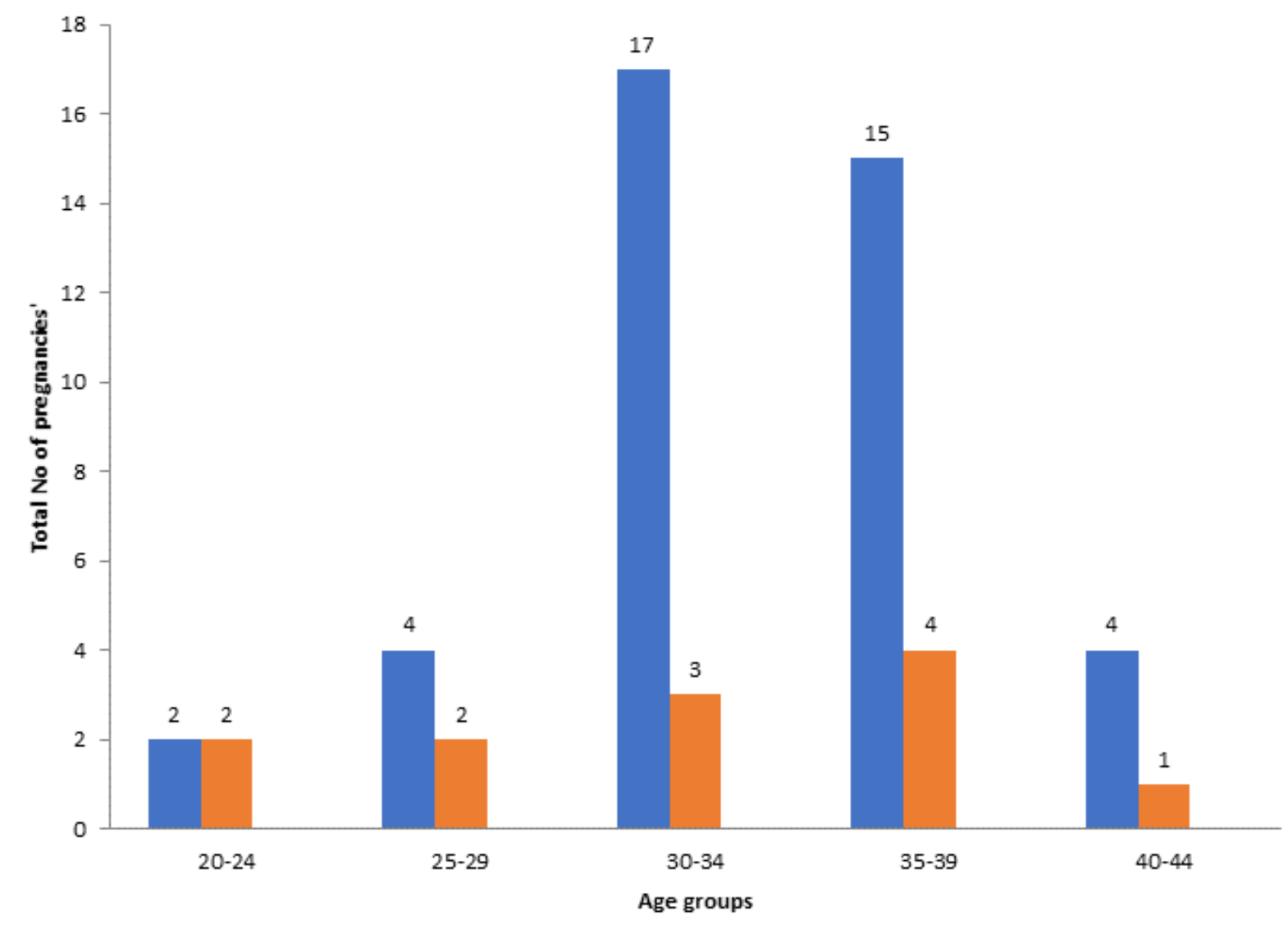

No of Patients

- Pregnancies 
Figure 2

Sensitivity and specificity of $E_{2}$ and $A M H$ in predicting pregnancy.

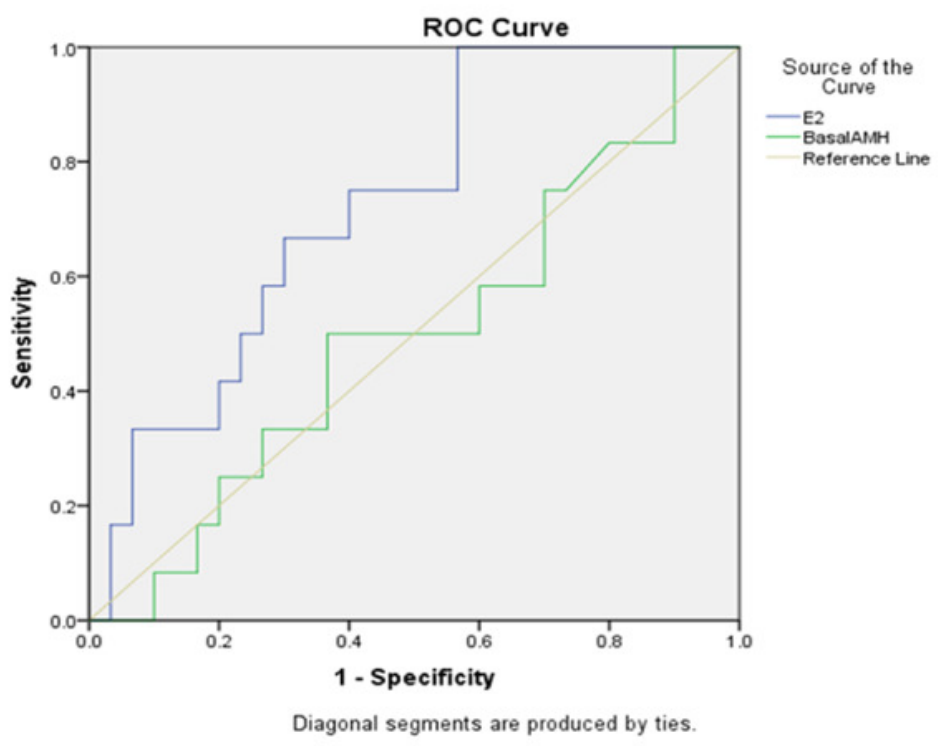


Table $\mathbf{1}$ (on next page)

AMH distribution in blood samples 
1

2

3

4

5

6 Table 1. AMH distribution in blood samples

7

8

9

10

11

12

13

14

15
AMH Blood Level Concentration Frequency \% Patients

$22 \quad 52.4$

17

3

52.4

40.5

$\leq 0.3-0.9 \mathrm{ng} / \mathrm{ml}$

7.1 
Table 2 (on next page)

Correlation between AMH and number of oocytes collected, matured, and fertilised during stimulation 
1

2

3

4 Table 2. Correlation between AMH and number of oocytes collected, matured, and fertilised

5 during stimulation

6

Total no. Total no. Total no.

of of of

oocytes oocytes oocytes $\% \quad \%$ oocytes

collected matured fertilized oocytes oocytes fertilized

\begin{tabular}{llllllll}
\hline & & \multicolumn{6}{c}{ collected } \\
\hline AMH & 81 & 62 & 69 & $65.4 \%$ & $60 \%$ & $61.6 \%$ \\
\hline $\begin{array}{l}\text { AMtegory } \\
\text { cateryal }\end{array}$ & 38 & 36 & 38 & $30.6 \%$ & $35 \%$ & $33.9 \%$ \\
\cline { 2 - 8 } & $\begin{array}{l}\text { Low to } \\
\text { Normal }\end{array}$ & 5 & 5 & 5 & $4.0 \%$ & $5 \%$ & $4.5 \%$ \\
\hline Total & & 124 & 103 & 112 & $100 \%$ & $100 \%$ & $100 \%$
\end{tabular}

7

8

9

10 
Table 3 (on next page)

Chi Square analysis of number of embryos transferred and $\mathrm{AMH}$ 
1

2

3

4 Table 3. Chi Square analysis of number of embryos transferred and AMH

5

\begin{tabular}{llll}
\hline & Value & $\begin{array}{l}\text { Degrees of Freedom } \\
(\mathrm{df})\end{array}$ & $\begin{array}{l}\text { Asymptotic } \\
\text { Significance (2-sided) }\end{array}$ \\
\hline Pearson Chi Square & $6.384^{\mathrm{a}}$ & 4 & .172 \\
Likelihood Ratio & 7.001 & 4 & .136 \\
N of Valid Cases & 42 & & \\
\hline
\end{tabular}

6

a. 7 cells (77.8\%) have expected count less than 5. The minimum expected count is 0.08

7

8

9 
Table 4 (on next page)

Chi Square analysis of AMH and day of embryo transfer 
1

2

3

4

5 Table 4. Chi Square analysis of AMH and day of embryo transfer.

\begin{tabular}{llll}
\hline & Value & $\begin{array}{l}\text { Degrees of Freedom } \\
(\mathrm{df})\end{array}$ & $\begin{array}{l}\text { Asymptotic } \\
\text { Significance (2-sided) }\end{array}$ \\
\hline Pearson Chi Square & $14.117^{\mathrm{a}}$ & 6 & 0.028 \\
Likelihood Ratio & 6.432 & 6 & 0.377 \\
N of Valid Cases & 42 & & \\
\hline
\end{tabular}

6

7

8

9 
Table 5 (on next page)

Pregnancy outcome and AMH category 
1

2

3

4

5

6 Table 5. Pregnancy outcome and AMH category

7

\begin{tabular}{llll}
\hline & \multicolumn{2}{l}{ Pregnancy Result } & \\
\hline AMH Category & Negative & Positive & Total \\
\hline High & 16 & 6 & 22 \\
Normal & 11 & 6 & 17 \\
Low Normal & 3 & 0 & 3 \\
Total & 30 & 12 & 42 \\
\hline
\end{tabular}

8

9

10 
Table 6(on next page)

Pearson correlation between basal $\mathrm{AMH}$ and $\mathrm{E}_{2}$, Age and FSH and oocytes 
1

2

3

4

5 Table 6. Pearson correlation between basal AMH and $\mathrm{E}_{2}$, Age and FSH and oocytes

6

\begin{tabular}{|c|c|c|c|c|c|c|c|}
\hline \multirow{7}{*}{} & & $\mathrm{E}_{2}$ & Age & FSH & $\begin{array}{c}\text { Number } \\
\text { of } \\
\text { oocytes }\end{array}$ & $\begin{array}{c}\text { Number of } \\
\text { mature } \\
\text { oocytes }\end{array}$ & $\begin{array}{c}\text { Number of oocytes } \\
\text { fertilized }\end{array}$ \\
\cline { 2 - 8 } & $\begin{array}{c}\text { Pearson } \\
\text { correlation } \\
\text { coefficient }\end{array}$ & 0.151 & -0.028 & - & 0.206 & 0.164 & 0.228 \\
& $\begin{array}{c}\text { Significance } \\
\text { value (2-tailed) }\end{array}$ & 0.341 & 0.859 & 0.240 & 0.191 & 0.300 & 0.146 \\
\cline { 2 - 8 } & $\begin{array}{c}\text { No. in the } \\
\text { sample }\end{array}$ & 42 & 42 & 42 & 42 & 42 & 42 \\
\hline \multirow{7}{*}{ Age } & $\begin{array}{c}\text { Pearson } \\
\text { Correlation }\end{array}$ & & & - & -0.271 & & \\
\cline { 2 - 8 } & $\begin{array}{c}\text { Significance } \\
\text { value (2-tailed) }\end{array}$ & & & 0.583 & 0.082 & & \\
\cline { 2 - 8 } & $\begin{array}{c}\text { No. in the } \\
\text { sample }\end{array}$ & & & 42 & 42 & & \\
\hline
\end{tabular}

7

8 
Table 7 (on next page)

Logistic regression analysis of the variables for the prediction of pregnancy 
2

3 Table 7. Logistic regression analysis of the variables for the prediction of pregnancy

4

\begin{tabular}{lllllll}
\hline & & $\begin{array}{l}\text { Standard } \\
\text { error } \\
\text { (S.E.) }\end{array}$ & Wald & $\begin{array}{l}\text { Degree of } \\
\text { Freedom } \\
(\mathrm{df})\end{array}$ & $p$-value & $\begin{array}{l}\text { OR=Exp } \\
(\mathrm{B})\end{array}$ \\
\hline $\mathrm{E}_{2}$ & .001 & .000 & 3.396 & 1 & .065 & 1.001 \\
$\mathrm{LH}$ & -.556 & .273 & 4.144 & 1 & .042 & .574 \\
Basal AMH & -.335 & .239 & 1.967 & 1 & .161 & .715 \\
Age & -.146 & .091 & 2.593 & 1 & .107 & .864 \\
Non-Fertilized & .150 & .368 & .166 & 1 & .683 & 1.162 \\
Basal FSH & -.102 & .136 & .559 & 1 & .455 & .903 \\
Constant & 4.451 & 3.452 & 1.663 & 1 & .197 & 85.744 \\
& & & & & &
\end{tabular}

$6 \quad E_{2}$, Estrogen; LH, luteinizing hormone; AMH, anti-Mullerian hormone; FSH, follicle stimulating 7 hormone. LH has $p=0.042(p<0.05)$ and $E_{2}$ has $p=0.065$, which is significant at a $10 \%$ level.

8 In this logistic regression model, the remaining variables are not significant (p-values $>0.10$ ).

9

10

11 
Table 8(on next page)

Chi Square analysis of Number of oocytes fertilized and $\mathrm{AMH}$ 
1

2

3

4

5 Table 8: Chi Square analysis of Number of oocytes collected and AMH.

\begin{tabular}{llll}
\hline & Value & $\begin{array}{l}\text { Degrees of Freedom } \\
(\mathrm{df})\end{array}$ & $\begin{array}{l}\text { Asymptotic } \\
\text { Significance (2-sided) }\end{array}$ \\
\hline Pearson Chi Square & $21.246^{\mathrm{a}}$ & 8 & .007 \\
Likelihood Ratio & 21.317 & 8 & .006 \\
N of Valid Cases & 42 & & \\
\hline
\end{tabular}

6

$7 \quad$ a 13 cells (86.7\%) have expected count less than 5. The minimum expected count is 0.12 
Table 9 (on next page)

Chi Square analysis of Number of oocytes collected and AMH 
1

2

3

4

5 Table 9. Chi Square analysis of Number of oocytes fertilized and AMH.

\begin{tabular}{llll}
\hline & Value & $\begin{array}{l}\text { Degrees of Freedom } \\
(\mathrm{df})\end{array}$ & $\begin{array}{l}\text { Asymptotic } \\
\text { Significance (2-sided) }\end{array}$ \\
\hline Pearson Chi Square & $18.504^{\mathrm{a}}$ & 12 & .101 \\
Likelihood Ratio & 17.736 & 12 & .124 \\
N of Valid Cases & 42 & & \\
\hline
\end{tabular}

6

$7 \quad{ }^{1} 18$ cells $(85.7 \%)$ have expected count less than 5. The minimum expected count is 0.02 